\title{
From stitch to society: a multi-level and participatory approach to design research
}

\section{Introduction}

The aims of this paper are twofold: to describe a doctoral research project that investigated the theme of openness in fashion, and to discuss a distinctive, practice-based approach to design research that emerged through this process. This approach uses the generative processes of designing and making, in collaboration with participants, to investigate research questions at multiple levels: from micro-scale practical challenges to much broader social issues.

The project in question explored the potential for opening and altering existing knitted fabrics while simultaneously investigating the role of homemade clothes in challenging the conventional fashion system; it also considered opportunities for amateur knitters to engage in creative design. A series of workshop activities supported the exploration of these areas of interest, with insights relating to each one emerging throughout the process. In this article I have revisited and extended my original contribution to the Research Through Design 2015 conference that discussed this research, ${ }^{1}$ adding commentary to frame the project more explicitly in terms of the multi-level approach. I will first describe the research context and activity in detail, before stepping outside this particular example to discuss the potential of this novel approach to design research.

\section{Background to the research}

The research considered amateur fashion making - which I describe as "folk fashion" - as a strategy for sustainability. The fashion system, as it currently stands, poses multiple challenges in terms of sustainability: negative environmental and social impacts occur in all phases of a garment's lifecycle. ${ }^{2}$ I launched my experimental knitwear label, Keep \& Share, in response to these challenges, investigating strategies of longevity and versatility. After a few years of running my practice I began to facilitate other people's making, running knitting workshops and projects in a range of contexts. These experiences sparked my curiosity regarding the relationship between folk fashion and sustainability.

\footnotetext{
${ }^{1}$ Amy Twigger Holroyd, "Re-knitting: exploring openness through design," in Proceedings of the $2^{\text {nd }}$ Biennial Research Through Design Conference, Cambridge, UK, March 25-7, 2015, Article 33, DOI: 10.6084/m9.figshare.1327993.

${ }^{2}$ Forum for the Future, Fashioning Sustainability: A Review of the Sustainability Impacts of the Clothing Industry (2007).
} 
My working understanding of sustainability is rather broader than the common definition that involves three interconnected elements: environment, society and economy. This conception has been criticised for its dependence on conventional economic thinking. ${ }^{3}$ As an alternative, Ehrenfeld offers a positive, aspirational definition: "the possibility that humans and other life will flourish on the Earth forever". $A$ focus on flourishing encourages us to understand human well-being as an integral element of sustainability; thus, thinking about fashion and sustainability must encompass the individual wearer's lived experience of the fashion system. My research started from the idea that folk fashion could overcome some of the environmental and social problems associated with conventionally produced clothing, and offer a more satisfying fashion experience. Indeed, both individual makers and sustainable design experts sometimes see homemade clothes as sustainable, in comparison with mass-produced "fast fashion". However, this view is largely based on a simplistic and romantic view of the homemade, which has received little critical examination. In order to consider whether making could offer a more sustainable alternative to the conventional fashion system, I identified the need to investigate makers' lived experiences of wearing homemade clothes in a culture dominated by mass-produced garments. This challenge connects me with researchers in a broad range of academic disciplines exploring theories around fashion, culture and craft.

Early in the project I became interested in the strong emphasis within knitting culture on making new items. Although the reworking of existing items would have been an integral element of knitting activity in the past, such practices have fallen out of favour in recent decades. This arguably restricts the advantages of knitting in terms of sustainability, as it mirrors - rather than challenges - the linear production-consumption model of the mainstream fashion industry. Gill and Lopes argue that too many sustainable design initiatives involve the production of new things; they suggest that "the challenge for the material practices of design might be recast in terms of a negotiation with those things already in existence". ${ }^{5}$ Excited by this idea, I chose to use my practice-based research to develop methods of reworking using knit-based techniques, skills and knowledge.

Two central themes can thus be identified within the research project: revitalising the practice of reknitting, and examining the potential of folk fashion to challenge the mainstream fashion system. The

\footnotetext{
${ }^{3}$ Tim Jackson, Prosperity Without Growth: Economics for a Finite Planet (London: Earthscan, 2009).

${ }^{4}$ John Ehrenfeld, Sustainability By Design: A Subversive Strategy for Transforming Our Consumer Culture (New Haven: Yale University Press, 2008): 49.

${ }^{5}$ Alison Gill and Abby Mellick Lopes, "On Wearing: A Critical Framework for Valuing Design's Already Made," Design and Culture, 3, no. 3: 312.
} 
former requires an engagement with the stitches that form knitted fabrics, and with the technical and practical challenges of remaking. The scope of the latter is much broader, requiring an exploration of a particular practice within contemporary society. Although these two frames of reference may seem rather disparate, I identified a conceptual thread that connected them: openness. On the micro level I was thinking about opening knitted fabrics; on the macro level I was thinking about opening the fashion system.

\section{Openness}

The trend of openness has reached many fields of life, creating movements such as open source software, open manufacturing and open gaming. ${ }^{6}$ In each area, we see the breaking down of hierarchical relationships between professional experts and amateur users; the role of the user is fundamentally altered from passive observer to active contributor. ${ }^{7}$ There is a clear link between making and openness. Openness, after all, relates to a "making and doing" culture, and an atmosphere of sharing and collaboration. The cultures and communities of amateur craft have offered an opportunity for people to actively create for centuries. Making culture is based on sharing, with activities such as knitting, quilting and embroidery drawing on a rich resource of traditional designs and an ethos of communal evolution. ${ }^{8}$ Open culture is vibrant in the world of knitting; knitters have embraced the potential of the internet for connecting and sharing their knowledge. A more practical interpretation of openness can be observed in the contemporary "maker movement".

Frustrated by the sealed units and proprietary tools used in electronic hardware, makers protest, "If you can't open it, you don't own it". ${ }^{9}$ This attitude corresponds with my ideas about opening and altering items of knitwear; however, unlike the physical restrictions encountered by makers seeking to tinker with electronic artifacts, the knitted structure is inherently open and capable of reconfiguration. Activity is instead limited by a lack of knowledge of how to open and alter the fabric, and cultural expectations of the ways in which we should interact with our clothing.

Shifting to the macro level, openness offers a useful means of thinking about alternatives to the current fashion system. I choose to think about fashion as a commons: a valuable resource, shared by a

\footnotetext{
${ }^{6}$ Michel Avital, "The Generative Bedrock of Open Design," in Open Design Now, ed. Bas van Abel, Lucas Evers, Roel Klaassen and Peter Troxler (Amsterdam: BIS Publishers, 2011): 48-58.

${ }^{7}$ David Gauntlett, Making Is Connecting, (Cambridge: Polity, 2011).

${ }^{8}$ Kirsty Robertson, 2010. "Embroidery Pirates and Fashion Victims: Textiles, Craft and Copyright," Textile: Journal of Cloth and Culture, 8, no. 1, 86-111.

${ }^{9}$ Mister Jalopy, "Owner's Manifesto," Make (2005). Link no longer working.
} 
community. Within this resource, I see all of the garments - new, old, fashionable, unfashionable - in existence. From a more conceptual viewpoint, I see every desirable way of appearing through dress, throughout history. Fashion depends on this broad, varied, vibrant resource: new fashions involve existing styles being revisited, recombined or recontextualised. I believe it is important for our well-being - and therefore sustainability - to have an open fashion commons, offering diverse options with which we can construct our identities. Some researchers argue that fashion is already an open commons, because it has minimal legal protections for its creative design. ${ }^{10}$ While I acknowledge that intellectual property law affects the openness of the commons, I feel that other factors are restricting access to, or enclosing, this resource.

As a maker, I am particularly interested in the role of manufacture as a mechanism of enclosure. Clothing production has become increasingly industrialised and professionalised in recent decades and thus wearers have become geographically and psychologically alienated from the making of their clothes. The businesses that produce the garments we wear restrict our use of the fashion commons because they make many choices about what is available and, as dependent wearers without an independent means of production, we can only choose from the options provided. This enclosure can be challenged; opening the fashion commons would involve gaining access to a greater diversity of styles, and making or adapting our own clothes is an accessible strategy for doing so. On the other hand, knitting is not as open as it may first appear. In my experience many amateurs have a desire to be "more creative", but lack the confidence to work without a pattern. Thus, while developing my thinking about the fashion system I encountered another angle on openness: opening design.

\section{Research process}

The entire research project is graphically represented in Figure 1. The three levels of enquiry discussed above - opening garments, opening design, and opening fashion - are communicated via three concentric bands; the starting points for each are summarised in the left-hand section. Stepping forward to the central section of the diagram, I will now discuss the means by which these three levels were empirically investigated.

${ }^{10}$ Christine Cox and Jennifer Jenkins, "Between the Seams, a Fertile Commons: An Overview of the Relationship Between Fashion and Intellectual Property," Ready to Share: Fashion and the Ownership of Creativity, USC Annenberg, January 29, 2005, http://www.learcenter.org/pdf/RTSJenkinsCox.pdf (accessed October 16, 2016). 
The research process was primarily guided by the micro-level challenge of opening knitted garments, developing and testing techniques for reworking by manipulating the individual stitches from which they are formed. This task provided a practical platform through which I also investigated openness in terms of the two other levels identified. Because existing garments are endlessly variable, conventional prescriptive patterns are of little use; makers must make their own technical and creative decisions. Therefore, in my research, reknitting offered an excellent opportunity to investigate the ability of amateurs to design for themselves. It also enabled me to investigate another aspect of opening design: exploring the creation of "open" resources to support amateur makers in this contingent and open-ended activity. The making activities supported empirical exploration at the macro level, too: by working closely with a group of knitters to develop and test the reknitting techniques, I was able to gain a deep insight into their experiences of making and wearing homemade clothes, and the ways in which these activities affect their relationship with the fashion system.

\section{RESEARCH PROCESS}

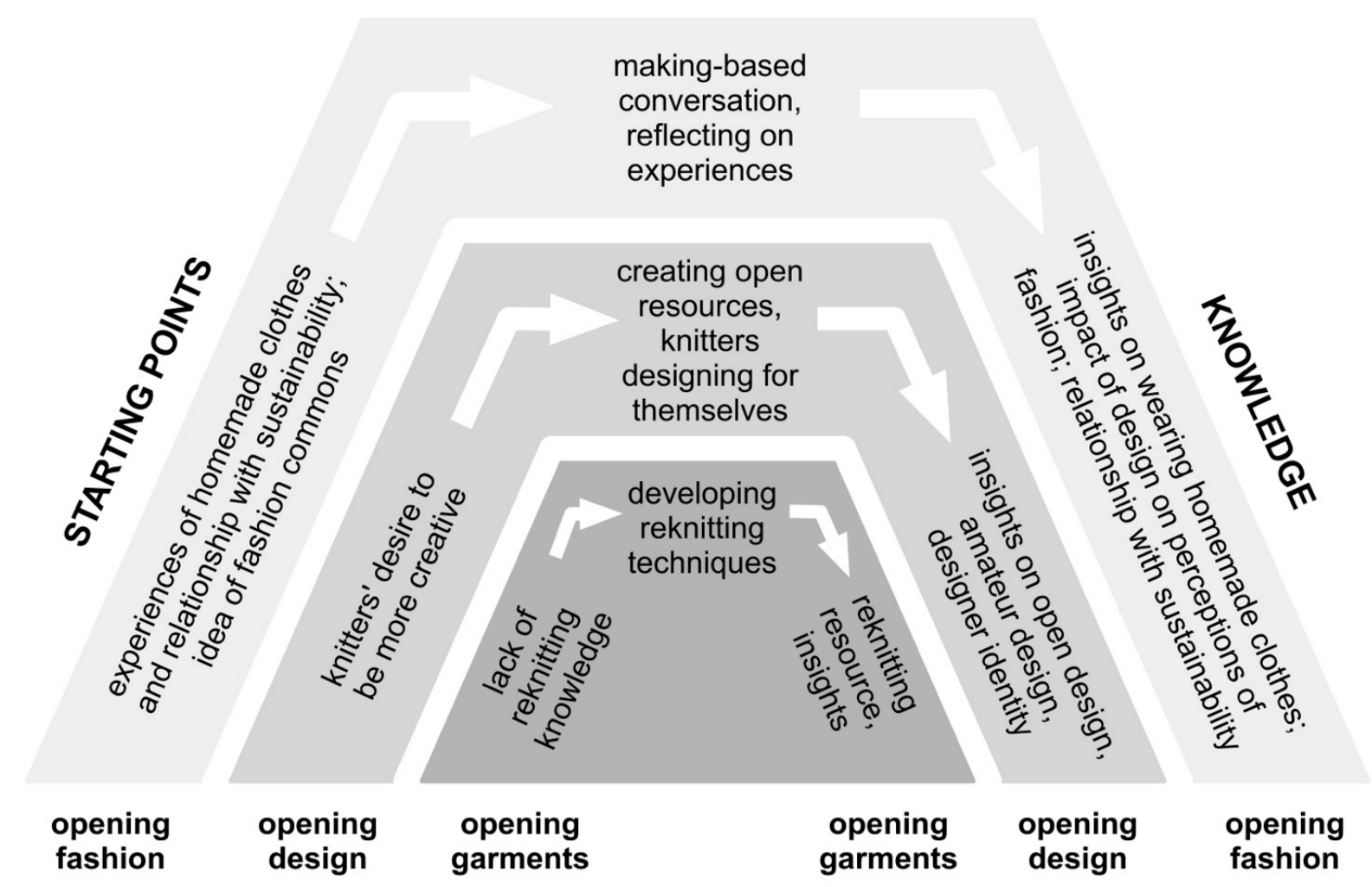

Figure 1. Summary of the reknitting-based design research project. (Amy Twigger Holroyd)

During the first phase of activity, I worked independently, developing the reknitting methods through iterative cycles of planning, sampling and reflection. I gradually worked through my own ideas about reknitting, researched methods from the past and built a spectrum of techniques that could be used to alter and rework any knitted garment - whether hand-knitted or mass-produced (Figure 2). Because I intended 
that these techniques would be used by amateur makers, I recruited six female amateur knitters, aged between 43 and 66, to take part in the project. The majority had previously attended one of my skills-based knitting workshops, and all were motivated to take part by the opportunity to explore design and creativity. The workshops I had carried out within my professional practice informed the approach I took to this research; I had found the workshop environment to be conducive to open conversation. I ran four day-long workshops spanning a period of four months. At the early workshops, I tested my reknitting techniques, design activities and instructional materials with the group. The sessions gradually shifted from these short structured activities to a more fluid studio environment, as the knitters developed ideas for their individual projects: designing and executing an alteration to a knitted item from their own wardrobe. Examples of these projects are shown in Figures 3 and 4. I also conducted individual garment-based interviews in advance of the group activities and carried out three evening "knitting circle" sessions, which provided opportunities for structured discussion and reflection.

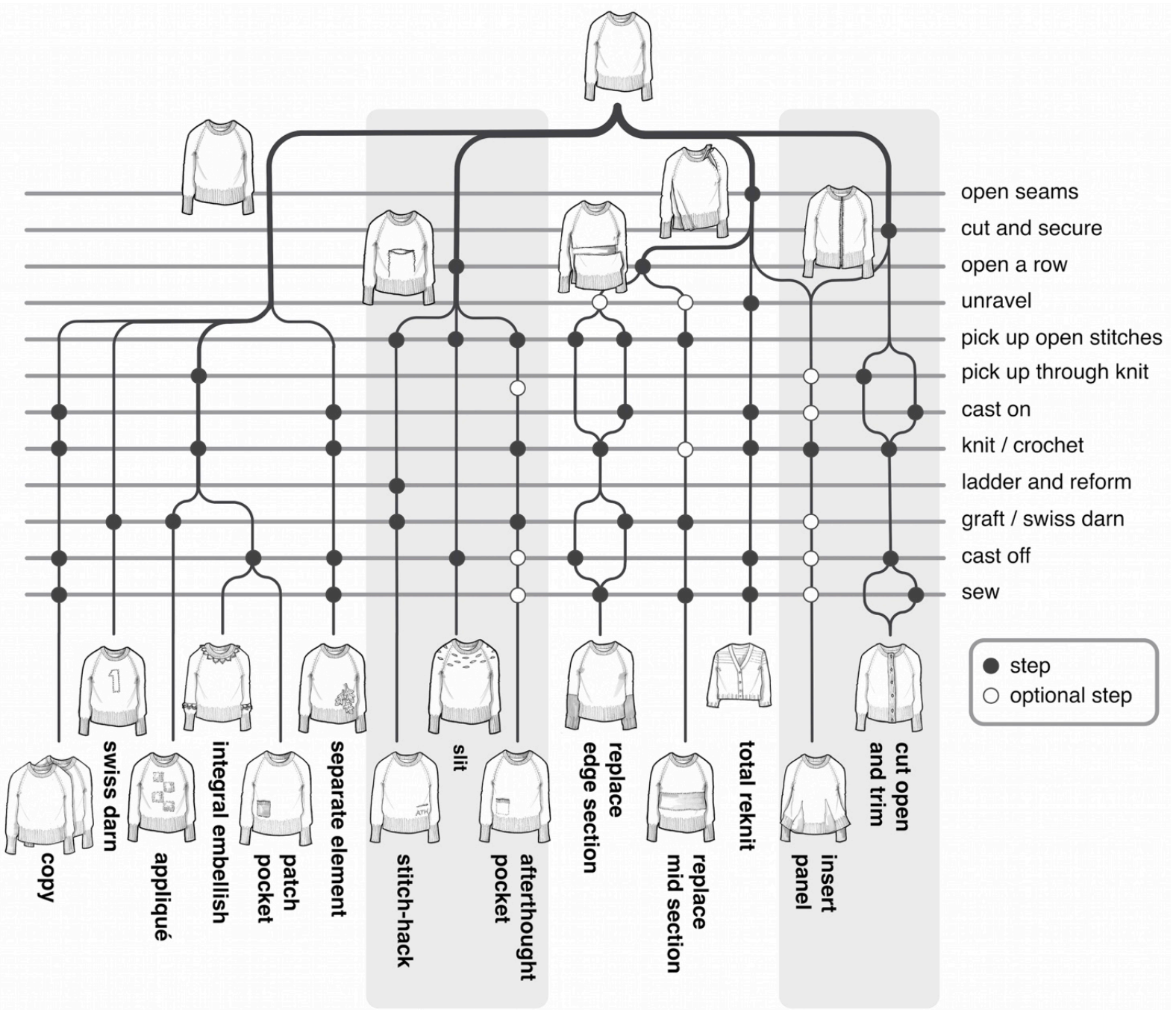

Figure 2. The spectrum of reknitting treatments. (Amy Twigger Holroyd) 


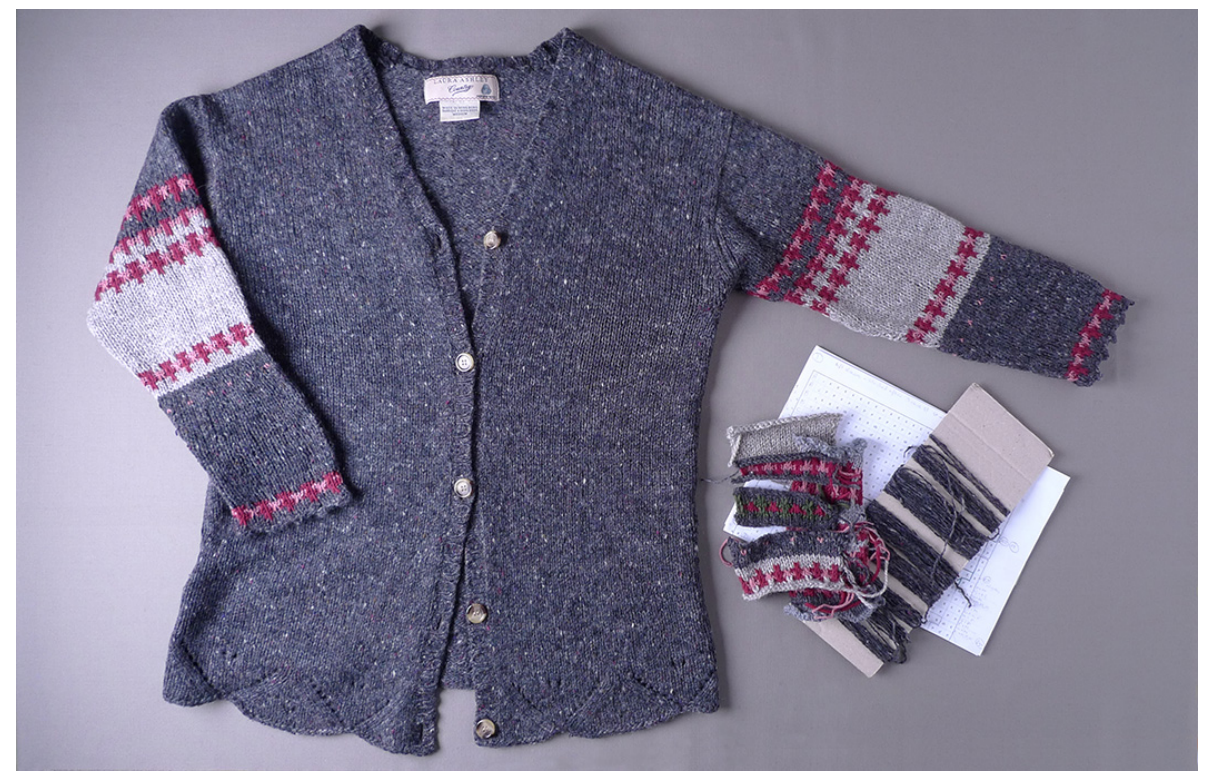

Figure 3. Kiki's reknitting project: replacement sleeves on a much-loved cardigan. (Amy Twigger Holroyd)

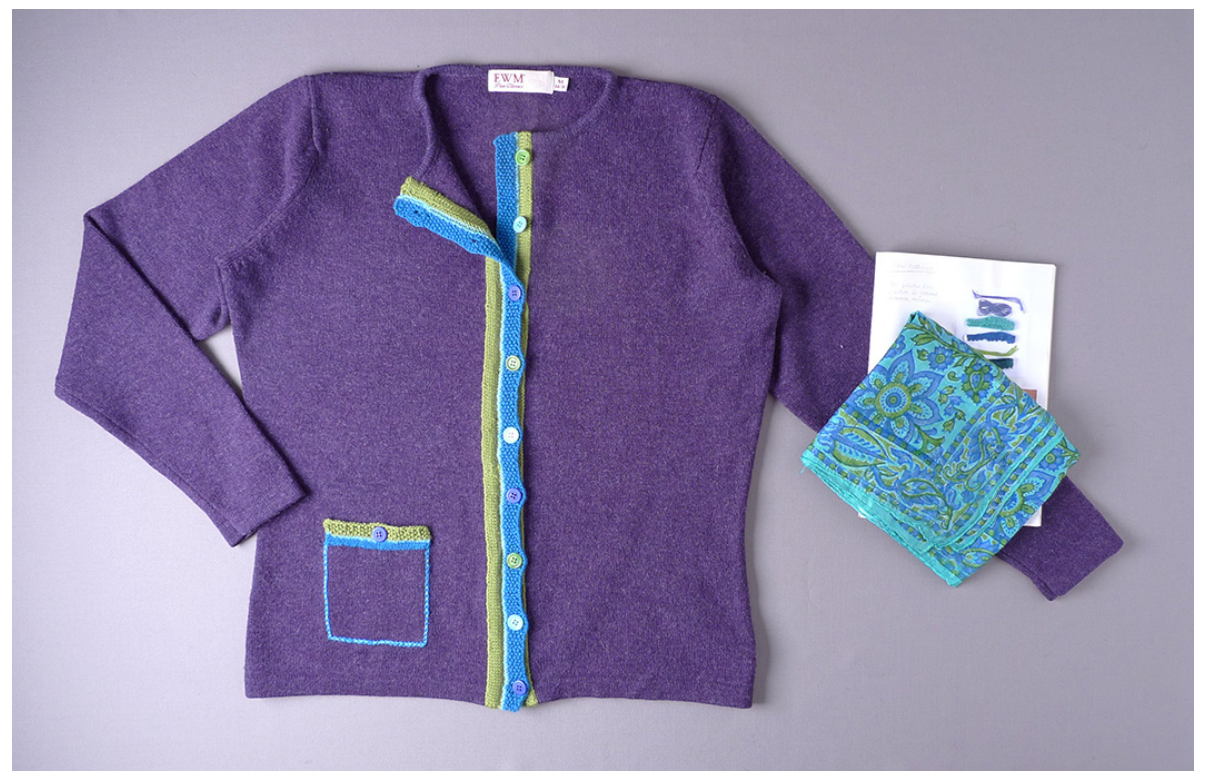

Figure 4. Julia's reknitting project: a "cardiganised" jumper with "afterthought pocket”. (Amy Twigger Holroyd)

All of the research activities were audio and video recorded, providing me with a wealth of raw data. By gathering data during the creative activity, rather than talking retrospectively, I was able to capture the participants' feelings first-hand as they tried out the reknitting techniques, and to examine the changes that occurred as the project progressed. I transcribed this data and used thematic coding to analyse it. ${ }^{11}$ In some cases the conversation directly addressed one of the levels of enquiry: the individual interviews, for example, focused on prior experiences of making and fashion, while the chat surrounding the early reknitting

${ }^{11}$ Colin Robson, Real World Research: A Resource for Users of Social Research Methods in Applied Settings, 3rd ed, (Chichester: Wiley, 2011). 
experiments was closely related to the task in hand. Most of the discussion within the group, however, jumped organically in terms of scale; thus, data relating to the three levels of enquiry was frequently intertwined. Conversations prompted by practical activities would range, from discussion of a specific technique, to comments about how it might be creatively applied, to reflections on its contemporary appeal. Through coding, I was able to isolate the conversation relevant to each level of enquiry and then, using a constant comparative method, sort the resultant data into themes and sub-themes. Thus, I discovered that the generative activity of making was well suited to the multi-level approach to design research that I had adopted. Active engagement in this creative process not only produced insights relevant to the immediate task, but also prompted spontaneous comments relating to the broader context.

\section{Findings}

As the right-hand section of Figure 1 indicates, the research generated knowledge relating to each of the levels of enquiry previously identified: opening garments, opening design, and opening fashion. At the level of opening garments, this knowledge comprised various outcomes relating to the practical process of reknitting, which I gathered together as an online reknitting resource. The resource includes step-by-step visual instructions for the treatments, advice to support the creative process, and a gallery of reknitting projects - such as the research participants' finished garments and a reknitting sampler garment that I produced (Figure 5). While the resource has received a positive response from the knitting community, at present it remains in prototype form, with further work needed to make the materials more user-friendly. This reflects the fact that in research through design projects, the goal is not the designed artifact (in this case, the reknitting resource), but the knowledge emerging from the process of development. 


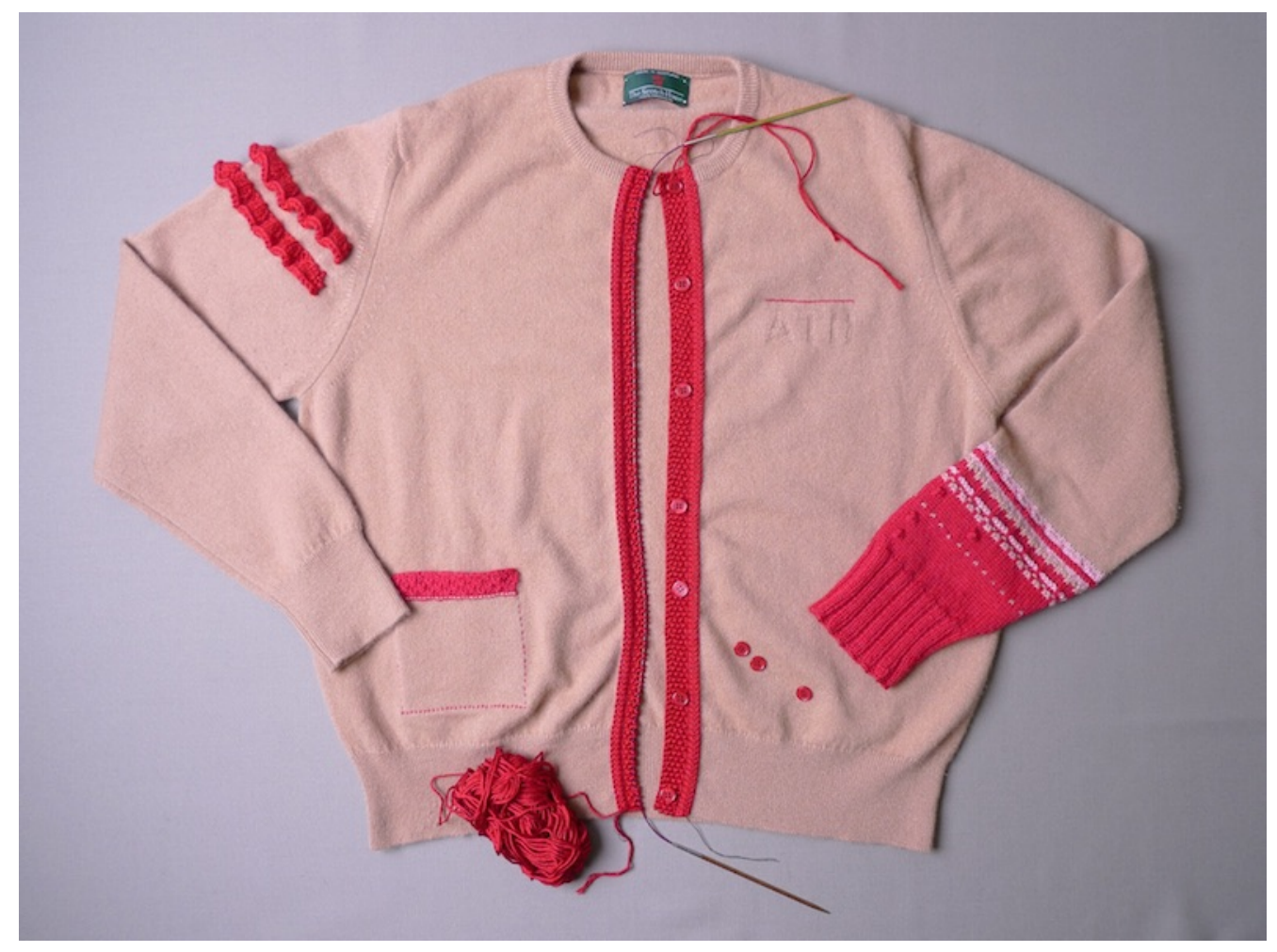

Figure 5. Reknitting sampler garment demonstrating five alterations. (Amy Twigger Holroyd)

The research generated insights relating to the reworking of existing items, and ways in which this can be supported; these insights have relevance beyond the immediate context of knitting. Firstly, I identified the need to be sympathetic to the material structures of the already-made, and to apply the in-depth knowledge we have as makers to the task of remaking. Secondly, the research demonstrated that we need to recognise the social and emotional aspects of remaking: that is, to understand the factors that affect what we perceive to be possible and desirable, and ways in which this perception can be altered. Thirdly, I found that a supportive culture must develop around remaking if it is to thrive, in order to foster a sense of shared practice and gradually build tacit knowledge.

The study also generated knowledge regarding the intermediate level of enquiry: opening design. This research shows that amateur knitters are able to design for themselves, and draw on their tacit knowledge when doing so. The key element in building the participants' abilities as designers was to create space for them to experiment and make creative decisions. I found that integrating peer support into this permissive space was key to its success; by discussing their ideas within the group, the knitters gained confidence in their decisions. The research also produced insights relating to the creation of open resources. As I developed the reknitting techniques I was keen to offer knitters as many options as possible, but was acutely aware that too much choice could stifle and close off activity just as much as a prescriptive knitting pattern. I came to see that open activity occupies a halfway point somewhere between the direct instructions of a conventional pattern and unsupported, endless choice. As de Mul observes, "creat[ing] order from 
disorder" in this manner is a meta-design challenge. ${ }^{12}$ Thus, during the project my role shifted from designermaker to meta-designer-maker. While my previous practice involved designing and making garments to sell, during this project I was designing fragments of knit processes, developing advice, and finding ways to present these resources. Furthermore, I was attempting to facilitate the creativity of others. This corresponds with von Busch's "hacktivist" designer role, which involves "designing material artefacts as well as social protocols". $^{13}$

Finally, I will return to the macro level to consider whether reknitting - and folk fashion more generally - can contribute to an open fashion commons. Through this research, I was able to build a nuanced understanding of the lived experience of wearing homemade clothes. While many people who make their own clothes find this to be an empowering, positive experience, others find amateur fashion making to be riddled with ambivalences, affecting the "success" of their homemade items. Yet making has great potential in terms of openness: I saw the participants in this research gain the confidence to access the fashion commons in ways that they would not have done previously. The activity contributed to their sense of well-being, and thus to a feeling of flourishing. Overall, I found that reknitting can be seen as an effective strategy for sustainability. It not only provides a means of extending product life, but more holistically offers an alternative means of participating in fashion, and a way of addressing the relationship between fashion and consumption.

\section{A transferable approach?}

In this project I explored a central theme at multiple levels through a single set of activities. This tidy explanation may seem surprising, given that much of my work revolves around material engagement, qualitative insights and "messy" embodied knowledge. The graphic representation shown in Figure 1 emerged only in response to the challenge of summarising a sprawling research project in the concise conference paper and presentation demanded by the Research Through Design conference. ${ }^{14}$ Furthermore, the diagram is not sufficient to communicate the detail of the research; instead, it provides a scaffold around which to tell a more nuanced story, as I have done in the earlier part of this paper. Overall, I see the multilevel diagram as a top-level summary of my qualitative analysis that provides, as Tesch describes, "an image

\footnotetext{
${ }^{12}$ Jos de Mul, "Redesigning Design," in Open Design Now, ed. Bas van Abel, Lucas Evers, Roel Klaassen and Peter Troxler (Amsterdam: BIS Publishers, 2011): 37.

${ }^{13}$ Otto von Busch, Fashion-able (Gothenburg: Camino, 2009): 63.

${ }^{14}$ Twigger Holroyd, "Re-knitting".
} 
that we can grasp as the 'essence', where we otherwise would have been flooded with detail and left with hardly a perception of the phenomenon at all."15

Although I created the diagram after the research was completed, the multi-level concept was not a retrospective solution; all of the elements were in place at an early stage of the research. I had the explicit intention of producing knowledge relating to the broad question of homemade clothes alongside the specifics of reknitting and design, and intended to do so through the use of participatory workshops. Zimmerman et al. explain that research through design "is seldom conducted with a declared intention of creating and building theory. This means that theory development is in many cases more of an afterthought than an intentional outcome."16 This chimes with my experience of the Research Through Design conference; while the presentations were fascinating and diverse, on many occasions I found myself yearning for more effective communication of the knowledge generated through the research activity. Reflecting on the experience of the conference, I realised that the approach I had developed supports the articulation of theory, by highlighting areas of interest and identifying the knowledge generated relating to each one. Furthermore, it supports transparency by connecting the knowledge generated with information on the methods used. I believe, therefore, that my approach may have value for future projects in diverse contexts. To explore its transferability, I will first compare it to related strategies for practicing research through design.

A key characteristic of my project was its agenda of social change: I was seeking to investigate alternatives to the current, consumption-intensive, fashion system. This type of agenda is common in design research. Sangiorgi and Scott explain that "Design work is increasingly moving well beyond the conventional concerns of form, function and style to address difficult social challenges". ${ }^{17}$ Zimmerman et al. point out that the generative process of design is a potent means of exploring these challenges, providing "a creative way of investigating what a potential future might be". ${ }^{18}$ I chose to work at multiple levels, from the practical challenge of reconfiguring the loops of a knitted garment through to the fashion system as a whole. Sangiorgi

\footnotetext{
${ }^{15}$ Renata Tesch, “Qualitative Research: Analysis Types and Software Tools”, (Basingstoke: Falmer, 1990).

${ }^{16}$ John Zimmerman, Erik Stolterman and Jodi Forlizzi, "An Analysis and Critique of Research through

Design: Towards a Formalization of a Research Approach," in Proceedings of the $8^{\text {th }}$ ACM Conference on

Designing Interactive Systems (DIS '10), (New York: ACM Press, 2010): 317.

${ }^{17}$ Daniela Sangiorgi and Kakee Scott, "Conducting Design Research in and for a Complex World," in The Routledge Companion to Design Research, ed. Paul A. Rodgers and Joyce Yee (Abingdon: Routledge,
} 2015): 114 .

${ }^{18}$ Zimmerman, Stolterman and Forlizzi, "An Analysis and Critique of Research through Design,": 313. 
and Scott suggest that a breadth of scope is inevitable when pursuing social change: "[designers] must contend with questions of system level change, even if working on smaller and more manageable manifestations of the wider scale phenomenon." ${ }^{19}$

Sangiorgi and Scott identify approaches to research that use design to provoke critical reflection at the systems level. Broadly speaking, these strategies have much in common with the approach I developed: I aimed to elicit critical reflection on the fashion system. In several of the examples that Sangiorgi and Scott describe, this elicitation is prompted by people observing a "critical design" artifact in a gallery setting. In others, people are asked to interact with and use an artifact or service in their everyday lives. As Koskinen et al. explain, such research "places design into a naturalistic setting. Researchers follow what happens to it in that context; how people and communities understand it, make sense of it, talk about it, and learn to use it., ${ }^{20}$ My approach is similar: I was interested to see how the amateur knitters I worked with would incorporate the reknitting techniques into their existing practices. Yet my research strategy was much more participatory, involving the knitters in the generative processes of designing the reknitting techniques and using these techniques to create their own unique projects. It was this creative and collaborative experience - rather than the use of a finished artifact or service - that prompted critical reflection. Thus, the approach I developed has more in common with the concept of "critical making".

Critical making, an approach developed by Matt Ratto, uses group making activities to explore the values associated with various technical innovations. As Ratto explains, "the ultimate goal of critical making experiences is not the evocative or pedagogical object intended to be experienced by others, but rather the creation of novel understandings by the makers themselves." ${ }^{21}$ One such project used Arduino software and hardware along with various other physical components to explore the social and technical dimensions of intellectual property. Likewise, my research used hands-on making as a key element of the creative experience. As I have discussed elsewhere ${ }^{22}$ the context of the practical workshop - where people are

\footnotetext{
${ }^{19}$ Sangiorgi and Scott, "Conducting Design Research in and for a Complex World,": 114.

${ }^{20}$ Ilpo Koskinen, Thomas Binder and Johan Redström, “Lab, Field, Gallery and Beyond," Artifact 2, no. 1: 51.

${ }^{21}$ Matt Ratto, "Critical Making," in Open Design Now, ed. Bas van Abel et al. (Amsterdam: BIS Publishers, 2011): 205 .

${ }^{22}$ Emma Shercliff and Amy Twigger Holroyd, "Making with others: working with textile craft groups as a means of research," Studies in Material Thinking 14 (2016), Paper 07, www.materialthinking.org/sites/default/files/papers/0176_SMT_V14_P07_FA.pdf (accessed October 16, 2016).
} 
making, side-by-side - generates open and illuminating conversation that is incredibly valuable for research. Ratto agrees: "the movement to the material world often seems to be accompanied by a less functionalist, more emotional and embodied reaction to the topics under construction/discussion." ${ }^{23}$

Zimmerman et al. indicate that research through design projects generate both "theory on design" (knowledge about how and why people design) and "theory for design" (knowledge that can improve the practice of design). The theory generated in the research projects they discuss occupies a relatively practical sphere: frameworks and guiding philosophies for use by designers, for example. In my research I similarly aimed to produce knowledge that would support design. Yet I was also aiming to prompt reflections, and therefore generate knowledge, at the systems level. While this knowledge may ultimately inform design, it primarily relates to qualitative human experience and thus connects with insights gained through work in other disciplines. The discoveries I made about the experience of wearing homemade clothes, for example, make a contribution to fashion theory: an area of scholarship incorporating work by anthropologists, sociologists, psychologists, geographers and cultural theorists. By using a multi-level approach, I was able to explore different levels of enquiry - from stitch to society - through a single series of activities, and to be explicit about the knowledge that was being generated.

\section{Future use}

Having discussed the distinctive characteristics of the multi-level and participatory approach to design research, the next challenge is outlining how it may be used to guide future projects. Clearly, this approach would not be appropriate for every design research project; it is suited to the investigation of social challenges via small-scale creative initiatives. In broad terms, this approach enables the researcher to study a situation and to explore the implementation of change within it, in collaboration with others. The participatory element is important: the creative projects need to involve people to whom the social issue is relevant, in order to elicit meaningful responses. Making activities will prompt the participants to share their thoughts about how the prototype initiative would fit into their lives, along with comments relating to the social context. At the same time the designer-researcher can experience and reflect on a novel design role.

The refined and simplified version of the multi-level diagram shown in Figure 6 summarises the principles of this approach. In this skeleton schematic, the making and design levels have been linked together, in recognition of the fact that these generative and creative activities are likely to be intertwined. At the outset of a project, a researcher would need to identify starting points at each level of enquiry: a social

\footnotetext{
${ }^{23}$ Ratto, "Critical Making,": 206.
} 
challenge in the outer band, and prompts for a design/making project in the inner bands. I found it useful to identify a concept - openness, in the case of the reknitting project - to connect these starting points and direct the design exploration. The researcher would then plan the research process: a central programme of hands-on making activities involving participants. While these activities would primarily address the design/making challenge, the conversation prompted by these creative tasks would also generate insights relevant to the societal level. The researcher would need to develop an effective means of capturing the conversations, such as audio and video recording. To analyse the data, they would need to sift and sort the participants' comments relating to each level of enquiry, and identify emergent themes and sub-themes within them. The neat structure communicated in the diagram will inevitably be challenged by open-ended research questions, engaged participants and qualitative data. Yet, in my experience, the concept of multilevel enquiry provides a thread that helps the researcher to navigate a complex process and emerge with transferable knowledge. This knowledge will include insights that can inform and guide design and making activities alongside theory relating to the social context.

\section{RESEARCH PROCESS}

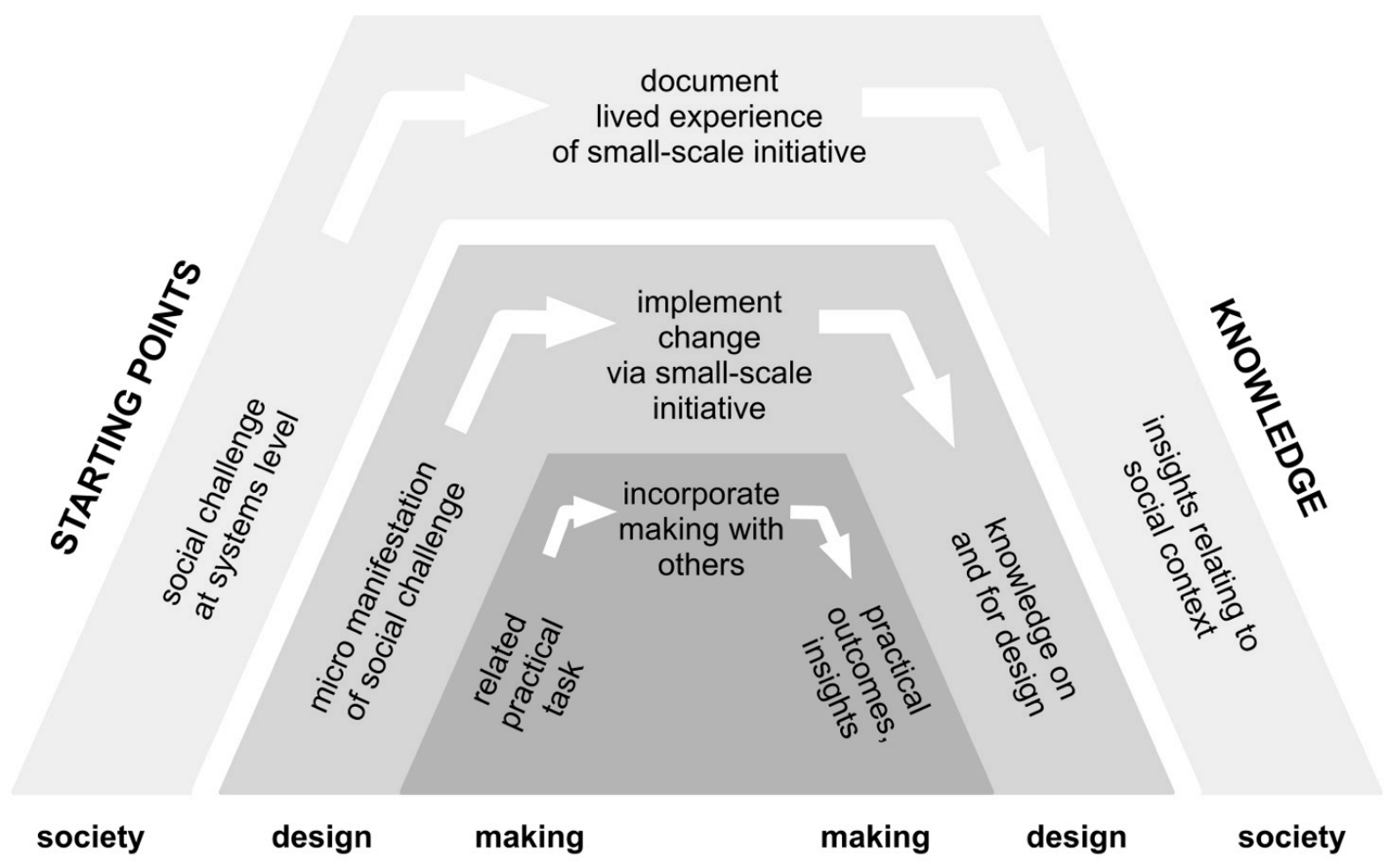

Figure 6. Summary of the multi-level and participatory approach to design research. (Amy Twigger Holroyd)

\section{Conclusion}

I have described a design research project that investigated openness in the context of fashion, generating insights relating to reworking, open design, and the lived experience of homemade clothes. According to 
Koskinen et al., "it is the theoretical scaffolding that makes the difference" between design practice and design research. ${ }^{24}$ Zimmerman et al. argue that the research through design community needs to rigorously document projects in terms of outcomes and methodology in order to highlight the transferable insights generated. ${ }^{25}$ The approach I developed uses a multi-level structure to underline the theoretical contribution of the research and indicate the methods used. I have found this to be an effective means of communication; by explicitly identifying the key contributions of my work, I make it easier for others to see connections with their own research interests and, where approriate, delve into the qualitative and material detail at the heart of the project.

This multi-level structure supported the planning and execution of my research project, as well as providing a scaffold to facilitate its dissemination. Thus, the approach could have value for future design research projects. The skeleton outline showing three levels of enquiry (making, design and society) passing through three stages (starting points, research process and knowledge) could be used to support diverse design-led research projects which address social issues through participatory hands-on making activities. Of course, any project involving people in the creative processes of designing and making will involve a great deal of complexity; this complexity will, by necessity, lie beneath the surface of the structure I have proposed. Furthermore, every design research project will have its own unique character, and a great deal of further work is required to maximise the adaptability of this approach. Yet I hope that the principle, and this example of its application, will be of use to others in the developing community of research through design practitioners.

\footnotetext{
${ }^{24}$ Koskinen, Thomas Binder and Johan Redström, “Lab, Field, Gallery and Beyond”: 47.

${ }^{25}$ Zimmerman et al., "An Analysis and Critique of Research Through Design”.
} 


\section{Biographical Statement}

Amy Twigger Holroyd is a designer, maker and researcher. Through her "craft fashion" knitwear label, Keep \& Share, she has explored the emerging field of fashion and sustainability since 2004. Her doctoral research at Birmingham Institute of Art \& Design informed her monograph, Folk Fashion: Understanding Homemade

Clothes (I.B.Tauris, 2017). As a postdoctoral Research Fellow at the University of Leeds she contributed to a collaborative project investigating the revitalisation of culturally significant designs, products and practices. In 2016 Amy joined Nottingham Trent University as a Senior Lecturer in Design, Culture \& Context. 


\section{Caption Sheet}

Figure 1. Summary of the reknitting-based design research project. (Amy Twigger Holroyd)

Figure 2. The spectrum of reknitting treatments. (Amy Twigger Holroyd)

Figure 3. Kiki's reknitting project: replacement sleeves on a much-loved cardigan. (Amy Twigger Holroyd)

Figure 4. Julia's reknitting project: a "cardiganised" jumper with "afterthought pocket". (Amy Twigger Holroyd)

Figure 5. Reknitting sampler garment demonstrating five alterations. (Amy Twigger Holroyd)

Figure 6. Summary of the multi-level and participatory approach to design research. (Amy Twigger Holroyd) 